\title{
Forecasting electricity load demand using hybrid exponential smoothing-artificial neural network model
}

\author{
Winita Sulandaria ${ }^{\mathrm{a}, \mathrm{b}, 1, *}$, Subanar $^{\mathrm{a}, 2}$, Suhartono $^{\mathrm{c}, 4}$, Herni Utami $^{\mathrm{a}, 4}$ \\ ${ }^{a}$ Universitas Gadjah Mada, Yogyakarta, Indonesia \\ ${ }^{b}$ Universitas Sebelas Maret, Surakarta, Indonesia \\ ${ }^{c}$ Institut Teknologi Sepuluh Nopember, Surabaya, Indonesia \\ ${ }^{1}$ winita@mipa.uns.ac.id*; ${ }^{2}$ subanar@ugm.ac.id; ${ }^{3}$ Suhartono@its.ac.id; ${ }^{3}$ herni_utami@ugm.ac.id \\ * corresponding author
}

Article history:

Received October 10, 2016

Revised December 3, 2016

Accepted December 3, 2016

\section{Keywords:}

Holt-Winter

Hybrid model

Exponential smoothing

Neural network

Electricity
Short-term electricity load demand forecast is a vital requirements for power systems. This research considers the combination of exponential smoothing for double seasonal patterns and neural network model. The linear version of Holt-Winter method is extended to accommodate a second seasonal component. In this work, the Fourier with time varying coefficient is presented as a means of seasonal extraction. The methodological contribution of this paper is to demonstrate how these methods can be adapted to model the time series data with multiple seasonal pattern, correlated non stationary error and nonlinearity components together. The proposed hybrid model is started by implementing exponential smoothing state space model to obtain the level, trend, seasonal and irregular components and then use them as inputs of neural network. Forecasts of future values are then can be obtained by using the hybrid model. The forecast performance was characterized by root mean square error and mean absolute percentage error. The proposed hybrid model is applied to two real load series that are energy consumption in Bawen substation and in Java-Bali area. Comparing with other existing models, results show that the proposed hybrid model generate the most accurate forecast.

Copyright $\odot 2016$ International Journal of Advances in Intelligent Informatics. All rights reserved.

\section{Introduction}

Electric power as source of energy is very important for human life, for industrial activities as well as in the daily life of the household. The distribution of electricity in Indonesia is handled by PLN (state-owned electric company). PLN supplies electricity needs of customers. There are four groups of customers: residential, industrial, commercial and public. The growth in population and industrialization means increasing number of customers and it should be translated into the growth in electricity demand. Since the electricity cannot be easily stored in large quantities to fulfill national demand, the electricity must be produced as much as is needed at all times. When the electricity supply is less than the demand, the consumer needs are not be served well. This can lead economic loss to the state. Otherwise, excess of the quantity of electrical energy supplied over its demanded resulting in wastage costs. Therefore, accurate model forecasting for electricity power load would be needed in development planning process of electric power system.

In this paper, univariate time series models are chosen by assuming that other variables can be captured in the load demand series itself. A time series of electricity load contains multiple seasonal cycles like daily, weekly and monthly seasonal cycles (see [1], [2], [3], [4], [5], and [6]). Over the years, several different forecasting techniques have been proposed to model the multiple seasonal electricity load. Some of them are based on the concept of exponential smoothing. Because of its simplicity, robustness and accuracy, Holt-Winter exponential smoothing became well known and was widely used to model single seasonal time series. Taylor [6] developed traditional Holt-Winter ([7]) to accommodate a second seasonal component. This method, called by double seasonal Holt-Winter 
(DSHW), corrects for residual using a simple autoregressive model. It assumes that the cycle of the intra-day is the same for all days of the week so that updates are the same for each day of the week. Gould et al. [4] improved DSHW by allowing the seasonal terms of one sub-cycle to be updated during the time for another sub-cycles. This approach is done by using the same sub-cycles for the days displaying similar patterns, such that it is possible with the model to have different smoothing parameters for different sub-cycles.

Several exponential smoothing methods are equivalent to point forecasts of special cases of linear innovation state space model ([8]). Exponential smoothing methods are optimal for a class of innovation state space models (see [9] and [10]). De Livera et al. [2] introduced an innovations state space modelling framework based on trigonometric formulation for exponential smoothing. This state space exponential smoothing model allowed for any autocorrelation in the residuals to be taken into account.

The existing exponential smoothing models ([3], [6], [11]) cannot capture nonlinearity. For handling nonlinear pattern, the model may need another nonlinear method. Artificial neural network (ANN) is a one of modern approaches that is widely used to model time series with nonlinearity (see, [12], [13], [14]). The implementation of ANN in modelling electricity data can be found in [15], [16], [17], [18], [19], [20], [21], and [22]. Since there probably exist linear and nonlinear patterns in load time series simultaneously, it is necessary to hybridize linear and nonlinear model for load demand forecasting.

In the recent study, some researchers hybridized two models to improve load forecasting performance. Sadaei et al. [23] and [24] implemented fuzzy as a part of hybrid models and [25] combined wavelet and ANN to forecast Australia electricity market load data. Shukur et al. [4] hybridized DSHW with ANN where components of DSHW are updated by substituting the original data with the output of ANN. In this paper, a hybrid approach based on exponential smoothing modified by [2] and neural network based on feed-forward back-propagation is proposed in order to enhance the forecasting accuracy. The strategy of building hybrid model used in this paper is different from those in [4]. In this study, state space exponential smoothing is proposed to determine the level, trend, seasonal and irregular components which then become the inputs of ANN.

\section{Brief Overview of Exponential Smoothing and Artificial Neural Network}

\section{A. Exponential Smoothing Model}

Forecasting methods based on the concept of exponential smoothing obtain the forecasts by combining the exponentially decreasing weighted past observations. In 1957, Holt introduced additive and multiplicative seasonal exponential smoothing ([26]). In the following year, [7] provided empirical test for Holt's methods and it then became more popular, known as Holt-Winters' method. Taylor [6] introduced DSHW as the extension of Holt-Winters method. This method is suitable for double seasonal time series with nested seasonal patterns and integer seasonality.

De Livera et al. [2] proposed exponential smoothing state space model with Box-Cox transformation, ARMA error, trend and trigonometric representation of seasonal components, named by TBATS. In this model, trigonometric functions based on Fourier series [27] is used to represent seasonal component. Thus, TBATS is more flexible approach to accommodate integer and non-integer seasonality, multiple nested and non-nested seasonal patterns.

A TBATS for double seasonal patterns has the form as follows.

$$
\begin{aligned}
& y_{t}^{(\omega)}= \begin{cases}\frac{y_{t}^{(\omega)}-1}{\omega} & \omega \neq 0 \\
\log y_{t} & \omega=0\end{cases} \\
& y_{t}^{(\omega)}=l_{t-1}+\phi b_{t-1}+\sum_{j=1}^{k_{1}} s_{j, t-1}^{(1)}+\sum_{j=1}^{k_{2}} s_{j, t-1}^{(2)}+d_{t}
\end{aligned}
$$

Level:

$$
l_{t}=l_{t-1}+\phi b_{t-1}+\alpha d_{t}
$$


Trend:

$$
b_{t}=(1-\phi) b+\phi b_{t-1}+\beta d_{t}
$$

First seasonal:

$$
\begin{aligned}
& s_{t}^{(1)}=s_{j, t-1}^{(1)} \cos \lambda_{j}^{(1)}+s_{j, t-1}^{*(1)} \sin \lambda_{j}^{(1)}+\gamma_{1}^{(1)} d_{t} \\
& s_{j, t}^{*(1)}=-s_{j, t-1}^{1} \sin \lambda_{j}^{(1)}+s_{j, t-1}^{*(1)} \cos \lambda_{j}^{(1)}+\gamma_{2}^{(1)} d_{t}
\end{aligned}
$$

Second seasonal:

$$
\begin{aligned}
& s_{t}^{(2)}=s_{j, t-1}^{(2)} \cos \lambda_{j}^{(2)}+s_{j, t-1}^{*(2)} \sin \lambda_{j}^{(2)}+\gamma_{1}^{(2)} d_{t} \\
& s_{j, t}^{*(2)}=-s_{j, t-1}^{2} \sin \lambda_{j}^{(2)}+s_{j, t-1}^{*(2)} \cos \lambda_{j}^{(2)}+\gamma_{2}^{(2)} d_{t}
\end{aligned}
$$

ARMA error:

$$
d_{t}=\sum_{i=1}^{p} \varphi_{i} d_{t-i}+\sum_{i=1}^{q} \theta_{i} \varepsilon_{t-i}+\varepsilon_{t}
$$

where $y_{t}^{(\omega)}$ is Box-Cox transformed observations with the parameter $\omega$ and $y_{t}$ is the observation at time $t$. The notation $l_{t}$ denotes the local level in period $t, b$ represents the long-run trend, $b_{t}$ is the short-run trend in period $t, s_{t}^{(1)}$ and $s_{t}^{(2)}$ are the first and the second seasonal components at time $t$ respectively, $d_{t}$ is an $\operatorname{ARMA}(p, q)$ process and $\varepsilon_{t}$ is a Gaussian white noise process with zero mean and constant variance $\sigma^{2}$. The notation $\phi$ is damped parameter and $\alpha, \beta, \gamma_{1}^{(1)}, \gamma_{2}^{(1)}, \gamma_{1}^{(2)}, \gamma_{2}^{(2)}$ are smoothing parameters. In seasonal equations, $s_{j, t-1}^{(i)}$ denotes the stochastic level and $s_{j, t-1}^{*(i)}$ is the stochastic growth in the level of the $i$ th seasonal components, $k_{i}$ indicates the number of harmonics for the $i$ th seasonal component, $\lambda_{j}^{(i)}=2 \pi j / m_{i}$ where $m_{i}$ is period of the $i$ th seasonal cycles for $i=$ 1,2 .

The argument of $\operatorname{TBATS}\left(\omega,\{p, q\}, \phi,\left\{\left\langle m_{1}, k_{1}\right\rangle,\left\langle m_{2}, k_{2}\right\rangle\right\}\right)$ indicates the Box-Cox parameter, ARMA parameter, damping parameter, and seasonal parameters. Distinguish from [28], in this case, all of the error sources are perfectly correlated, known as single source of error (SSOE). State space with SSOE is known as innovation state space. Innovation state space model for TBATS [2] for double seasonal cycles can be written as

$$
\begin{aligned}
& y_{t}^{\omega}=w^{\prime} x_{t-1}+\varepsilon_{t} \\
& x_{t}=F x_{t-1}+g \varepsilon_{t} .
\end{aligned}
$$

When all of the components of the model are present, it can be stated that $w^{\prime}=(1, \phi, a, \varphi, \theta)$,

$$
\begin{gathered}
x_{t}=\left(l_{t}, b_{t}, s_{t}^{(1)}, s_{t}^{(2)}, d_{t}, d_{t-1}, \ldots, d_{t-p+1}, \varepsilon_{t}, \varepsilon_{t-1}, \ldots, \varepsilon_{t-q+1}\right)^{\prime}, a=\left(a^{(1)}, a^{(2)}\right), a^{(1)}=\left(\mathbf{1}_{k_{1}}, \mathbf{0}_{k_{1}}\right) \\
a^{(2)}=\left(\mathbf{1}_{k_{2}}, \mathbf{0}_{k_{2}}\right), \varphi=\left(\varphi_{1}, \varphi_{2}, \ldots, \varphi_{p}\right), \theta=\left(\theta_{1}, \theta_{2}, \ldots, \theta_{p}\right), g=\left(\alpha, \beta, \gamma, 1,0_{p-1}, 1,0_{q-1}\right)^{\prime}, \gamma= \\
\left(\gamma^{(1)}, \gamma^{(2)}\right), \gamma^{(1)}=\left(\gamma_{1}^{(1)}, \gamma_{2}^{(1)}\right), \gamma^{(2)}=\left(\gamma_{1}^{(2)}, \gamma_{2}^{(2)}\right), \gamma_{1}^{(1)}=\gamma_{1}^{(1)} \mathbf{1}_{k_{1}}, \gamma_{2}^{(1)}=\gamma_{2}^{(1)} \mathbf{1}_{k_{1}} . F \text { is a matrix } \\
F=\left[\begin{array}{ccccc}
1 & \phi & 0_{\tau} & \alpha \varphi & \alpha \theta \\
0 & \phi & 0_{\tau} & \beta \varphi & \beta \theta \\
0^{\prime}{ }_{\tau} & 0^{\prime}{ }_{\tau} & A & B & C \\
0 & 0 & 0_{\tau} & \varphi & \theta \\
0_{p-1}^{\prime} & 0_{p-1}^{\prime} & O_{p-1, \tau} & I_{p-1, p} & O_{p-1, q} \\
0 & 0 & 0_{\tau} & 0_{p} & 0_{q} \\
0_{q-1}^{\prime} & 0_{q-1}^{\prime} & O_{q-1, \tau} & O_{q-1, p} & O_{q-1, q}
\end{array}\right],
\end{gathered}
$$


where $0_{\tau}=(0,0, \ldots, 0)$ be row vectors of length $\tau=2\left(k_{1}+k_{2}\right) . O_{u, v}$ is a $u \times v$ zero matrix, $I_{u, v}$ is a $u x v$ identity matrix, $B=\gamma^{\prime} \varphi, C=\gamma^{\prime} \theta, A=\left[\begin{array}{cc}A_{1} & O_{k_{1}, k_{2}} \\ O_{k_{2}, k_{1}} & A_{2}\end{array}\right], A_{i}=\left[\begin{array}{cc}C^{(i)} & S^{(i)} \\ -S^{(i)} & C^{(i)}\end{array}\right]$, where $C^{(i)}$ is $k_{i} x k_{i}$ diagonal matrices with elements $\cos \left(\lambda_{j}^{(i)}\right)$ and $S^{(i)} k_{i} x k_{i}$ diagonal matrices with elements $\sin \left(\lambda_{j}^{(i)}\right)$ for $j=1,2, \ldots, k_{i}$ and $i=1,2$. The parameters of TBATS can be obtained by maximum likelihood incorporating the least square criterion [2].

\section{B. Artificial Neural Network}

ANN can be used for modelling and forecasting nonlinear time series ([29], [30]). ANN learns from past behavior and finds subtle functional relationships among data in the past and then obtains forecast values via prediction future behavior. The structure of ANN consists of input nodes, hidden layers and output nodes. One or two hidden layers are enough for most forecasting problems [31]. The best network is obtained from the right combination of the number of input nodes, hidden layers and output nodes. This is also influenced by the weights that connecting every two nodes. Backpropagation is the most popular learning algorithm in selecting the weights. In its implementation, there are several optimization algorithm that can be included into the back-propagation algorithm, such as conjugate gradient, Gauss-Newton, and Levenberg-Marquardt. These algorithms can be used to train the network, finding the weights by minimizing the sum of square error function ([32]).

In this study, the network involves some inputs and one output. The input nodes are the past observations and the output is the forecast value. ANN model is focused on the feed forward neural network with Levenberg-Marquardt back-propagation algorithm.

\section{Proposed Hybrid Model and Algorithm}

Based on (1), the forecast values of TBATS are obtained from the summation of level, trend and seasonal components. Since there may be linearity and nonlinearity in load data series together, hybrid TBATS-ANN is proposed to improve forecasting performance. In this paper, TBATS is used to decompose load time series data into level, trend, seasonal and irregular components and ANN is applied to capture nonlinearity in the data. The idea is finding forecast value by using ANN where the past $m$ time period level, trend, seasonal and irregular obtained from TBATS be inputs of ANN. Framework of this study is shown in Fig. 1.

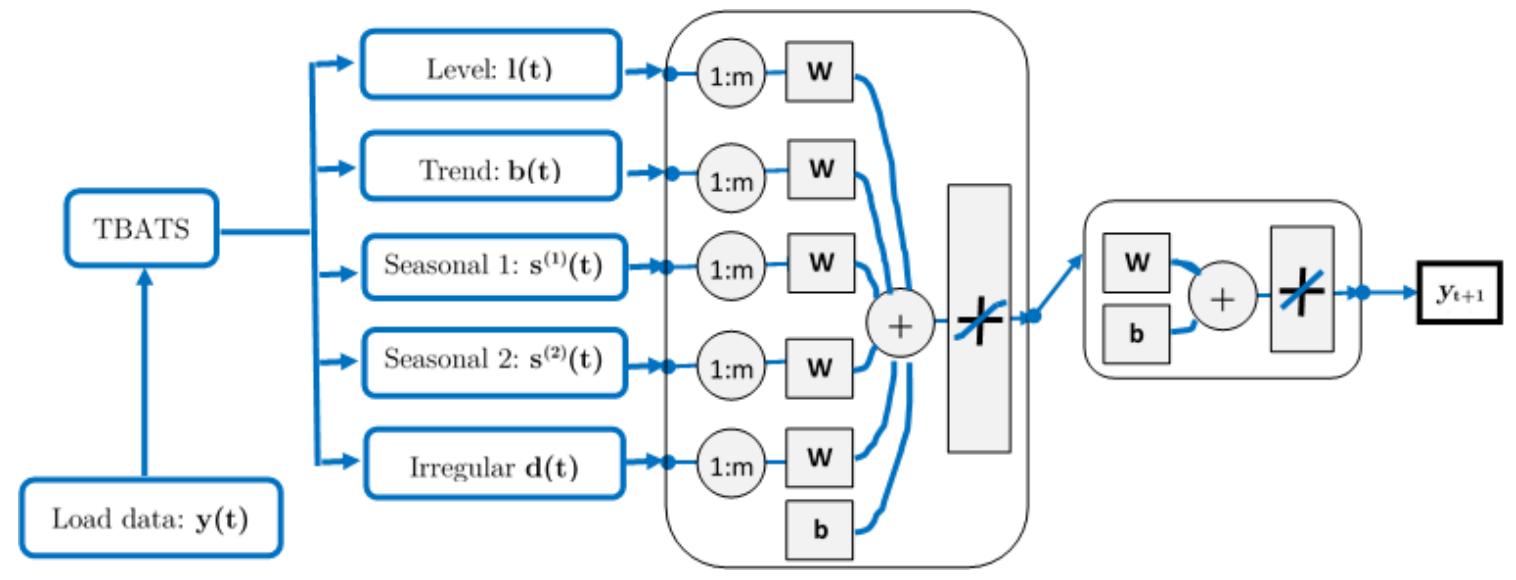

Fig. 1.Framework hybrid $\operatorname{TBATS}\left(\omega,\{p, q\}, \phi,\left\{\left\langle m_{1}, k_{1}\right\rangle,\left\langle m_{2}, k_{2}\right\rangle\right\}\right)-\operatorname{ANN}\left(m-n_{\text {hid }}-1\right)$.

Algorithm of the proposed method is presented below.

Step 1: Divide the data into in-sample and out-sample data.

Step 2: Apply TBATS model to decompose load time series which has complex seasonal into level, trend, seasonal and irregular components. The following steps show how TBATS model is constructed and how the model extract the datasets [2].

a. Estimate an initial value for Box-Cox parameter and transform the data. 
b. Estimate the period of seasonal cycles $\left(m_{1}\right.$ and $\left.m_{2}\right)$ based on the plot and autocorrelation function of the series.

c. Find seasonal component by first letting $m^{*}=\left\lfloor\max \left(m_{1}, m_{2}\right)\right\rfloor$, computing a $2 \times m^{*}$ moving average through the first few seasons of transformed data and then denoting this by $\left\{f_{t}\right\}$ for $t=$ $\frac{m^{*}}{2}+1, \frac{m^{*}}{2}+2, \ldots$. Let $z_{t}$ be the seasonal component, then $z_{t}=y_{t}^{(\omega)}-f_{t}$.

d. Approximate $z_{t}$ using $z_{t} \approx \sum_{i=1}^{2} \sum_{j=1}^{k_{i}} a_{j}^{(\mathrm{i})} \cos \left(\lambda_{j}^{(\mathrm{i})} t\right)+b_{j}^{(\mathrm{i})} \sin \left(\lambda_{j}^{(1)} t\right)$, and define the initial seasonal state estimates as $\hat{z}_{t}^{(i)}=\sum_{j=1}^{k_{i}} \hat{a}_{j}^{(\mathrm{i})} \cos \left(\lambda_{j}^{(\mathrm{i})} t\right)+\hat{b}_{j}^{(\mathrm{i})} \sin \left(\lambda_{j}^{(1)} t\right)$ where $\hat{a}_{j}^{(\mathrm{i})}$ and $\hat{b}_{j}^{(\mathrm{i})}$ are estimated by regressing $z_{t}$ against the trigonometric terms. The number of harmonics $k_{i}$ in the trigonometric models is selected based on the value of Akaike Information Criterion (AIC).

e. Approximate the initial level and trend components by computing a linear regression on the first $m^{*}$ deseasonalized values, against a time variable $t=1, \ldots, m^{*}$. The intercept and the slope of this regression are then set as the initial level and the initial trend respectively.

f. Optimize the initial state values along with the Box-Cox parameter and the smoothing parameters by maximizing the likelihood.

g. Select the best model based on the value of AIC. In this case, ARMA $(p, q)$ error component is only retained if the resulting model has lower AIC than the model with no ARMA component.

h. Obtain the level component by $l_{t}$, trend component by $b_{t}$, and the first and the second seasonal components by $s_{t}^{(1)}$ and $s_{t}^{(2)}$ respectively. Irregular component, $d_{t}$, is obtained from $y_{t}-l_{t}-$ $b_{t}-s_{t}^{(1)}-s_{t}^{(2)}$.

Step 3: Find ANN model with level, trend, seasonal and irregular components found at the second step as the inputs of the model. Inputs of ANN (see Figure 1) are $\mathbf{l}(\mathbf{t})=\left(l_{t-1}, l_{t-2}, \ldots, l_{t-m}\right), \mathbf{b}(\mathbf{t})=$ $\left(b_{t-1}, b_{t-2}, \ldots, b_{t-m}\right), \mathbf{s}^{(\mathbf{1})}(\mathbf{t})=\left(s_{t-1}^{(1)}, s_{t-2}^{(1)}, \ldots, s_{t-m}^{(1)}\right), \mathbf{s}^{(2)}(\mathbf{t})=\left(s_{t-1}^{(2)}, s_{t-2}^{(2)}, \ldots, s_{t-m}^{(2)}\right)$, and $\mathbf{d}(\mathbf{t})=$ $\left(d_{t-1}, d_{t-2}, \ldots, d_{t-m}\right)$. Let $m$ is chosen between 1 to $\min \left(m_{1}, m_{2}\right)$. In this study, the activation function in hidden layer is tangent sigmoid, which has the form $f(y)=\frac{1-e^{-2 y}}{1+e^{-2 y}}$, and activation function in output layer is linear function, $f(y)=y$. Back-propagation learning algorithm based on Levenberg Marquardt algorithm is used to estimate the weight $\mathbf{w}$ and the bias $\mathbf{b}$.

Step 4: Calculating forecast accuracy

Step 5: Comparing forecast performance using the root mean square error (RMSE) and mean absolute percentage error (MAPE) of TBATS, ANN, DSHW and TBATS-ANN.

\section{Illustration}

This research uses two load time series data for showing the forecasting performance of the proposed hybrid approach. A half-hourly load of Bawen, Salatiga, which is recorded from 1 February to April 2013 is used as the first illustration and an hourly load of Java-Bali from January until July 2010 be the second illustration. The forecast accuracy of proposed hybrid model is compared with TBATS, ANN, and DSHW. In this study, TBATS and DSHW are implemented using R.3.3.0 and ANN model is obtained through Matlab R2015a.

\section{A. Application to A Half Hourly Load Data}

A half-hourly load of Bawen substation measured in Megawatt (MW) taken from PT PLN P3B Java Bali Region Salatiga is used in this research. The three months data recorded from 1 February to 30 April 2010 is divided into training data set (in-sample) and testing data set (out-sample). The first two months is considered as an in-sample data and the rest month be out-sample data. The load series contains a daily and weekly seasonal patterns with period 48 and 336, respectively. Based on the selection procedure, TBATS does not need Box-Cox transformation and ARMA $(3,2)$ process is appropriate to describe the irregular component. Since there is no damping parameter, the growth rate $b_{t}$ was omitted from the model. Thus, the inputs of ANN are level, seasonal 1, seasonal 2 and irregular components of TBATS. In this case, the number of nodes in hidden layer is set to 10 and $m$ is chosen between 1 and $\min \left(m_{1}, m_{2}\right)=48$ so that the model produces the smallest MSE. The comparisons of forecast accuracy among the models are shown in Table 1 and Fig. 2. 
Table 1. Comparison of MAPEs and RMSEs of The Proposed Hybrid Approach with TBATS, ANN and DSHW for Load of Bawen

\begin{tabular}{lcccc}
\hline & \multicolumn{2}{c}{ In-sample } & \multicolumn{2}{c}{ Out-sample } \\
\cline { 2 - 5 } \multicolumn{1}{c}{ Methods } & $\boldsymbol{M A P E}$ & $\boldsymbol{R M S E}$ & $\boldsymbol{M A P E}$ & $\boldsymbol{R M S E}$ \\
\hline & & & & \\
TBATS $(1,\{3,2\},-,\{<48,5>,<336,5>\})$ & $3.2848 \%$ & 1.1261 & $4.1650 \%$ & 1.3211 \\
ANN $(48-10-1)$ & $0.9325 \%$ & 0.3624 & $1.3818 \%$ & 0.5272 \\
DSHW(48,336) & $0.8726 \%$ & 0.4170 & $0.9583 \%$ & 0.3647 \\
Proposed hybrid approach: & & & & \\
TBATS(1, $\{3,2\},-,\{<48,5>,<336,5>\})-A N N(48-10-1)$ & $0.8934 \%$ & 0.3765 & $0.9077 \%$ & 0.3155 \\
\hline
\end{tabular}

It can be seen from Table 1 that among the three single models, TBATS yields the highest RMSE and MAPE both in in-sample and out-sample and DSHW produces the smallest one. This probably because DSHW is designed to model integer and nested seasonal pattern which coincides in the given illustration while TBATS is more powerful in non-integer and non-nested seasonal pattern. By combining with ANN, the better performance of forecasting is achieved. Fig. 2 shows that hybrid TBATS $(1,\{3,2\},-,\{<48,5>,<336,5>\})$-ANN(48-10-1) yields the smallest MAPE and RMSE for the load of Bawen. It reduces MAPE of TBATS $77 \%$ and ANN $45 \%$ at out-sample.
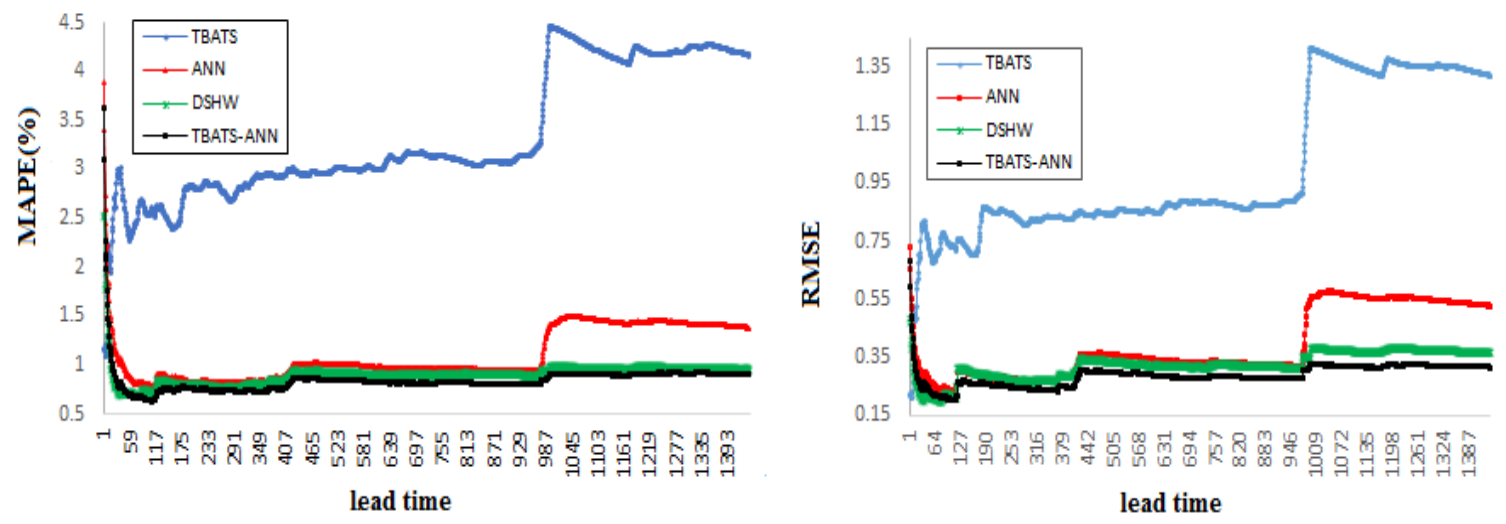

Fig. 2. Out-sample results of TBATS $(1,\{3,2\},-,\{<48,5>,<336,5>\}), \operatorname{ANN}(48-10-1)$, DSHW(48,336) and hybrid TBATS $(1,\{3,2\},-,\{<48,5>,<336,5>\})-\operatorname{ANN}(48-10-1)$ for the load of Bawen substation for April 2013.

\section{B. Application to An Hourly Load Data}

Java Bali load data used in the second illustration was taken from PT PLN P3B Java Bali for period 1 January 2010 to 31 July 2010. The data from January until June 2010 is used as in-sample and July 2010 as out-sample. Different from the first case, Java Bali load exhibits daily seasonal pattern with period 24 and weekly seasonal pattern with period 168. TBATS decomposed the series into level, trend, seasonal 1, seasonal 2 , and irregular components. Though the irregular component of the series is not uncorrelated, it cannot be described by ARMA model. This may have caused the MAPE and RMSE of TBATS relatively large. Comparisons of forecast accuracy between the selected TBATS, ANN, DSHW and proposed hybrid approach is presented in Table 2.

Table 2. Comparison of MAPEs and RMSEs of The Proposed Hybrid Approach with TBATS, ANN and DSHW for Load of Java Bali

\begin{tabular}{lcccc}
\hline \multirow{1}{*}{ Methods } & \multicolumn{2}{c}{ Training } & \multicolumn{2}{c}{ Testing } \\
& MAPE & RMSE & MAPE & RMSE \\
\hline TBATS $(1,\{0,0\}, 0.812,\{\langle 24,11>,<168,6>\})$ & 1.3765 & 253.7747 & 1.8718 & 330.2974 \\
ANN $(24-10-1)$ & 1.2340 & 235.7339 & 1.2426 & 241.9728 \\
DSHW $(24,168)$ & 0.8253 & 166.4451 & 0.9970 & 193.3964 \\
Proposed hybrid approach: & & & & \\
TBATS $(1,\{0,0\}, 0.812,\{<24,11>,<168,6>\})-A N N(24,10,1)$ & 0.7003 & 133.3449 & 1.0030 & 188.4585 \\
\hline
\end{tabular}


Generally, the proposed hybrid approach gives the best forecast performance. Based on MAPE and RMSE of out-sample presented in Figure 3, it can be concluded that forecasting performance obtained from $\operatorname{TBATS}(1,\{0,0\}, 0.812,\{\langle 24,11\rangle,<168,6\rangle\})$-ANN $(24,10,1)$ is more stable and better than other three single models. It also can be seen that the RMSE of TBATS, ANN and DSHW increased starting from the lead time 215, due to the Isra' Mi'raj religious holiday. It probably because of the inability of the three single models to capture the holiday effect.
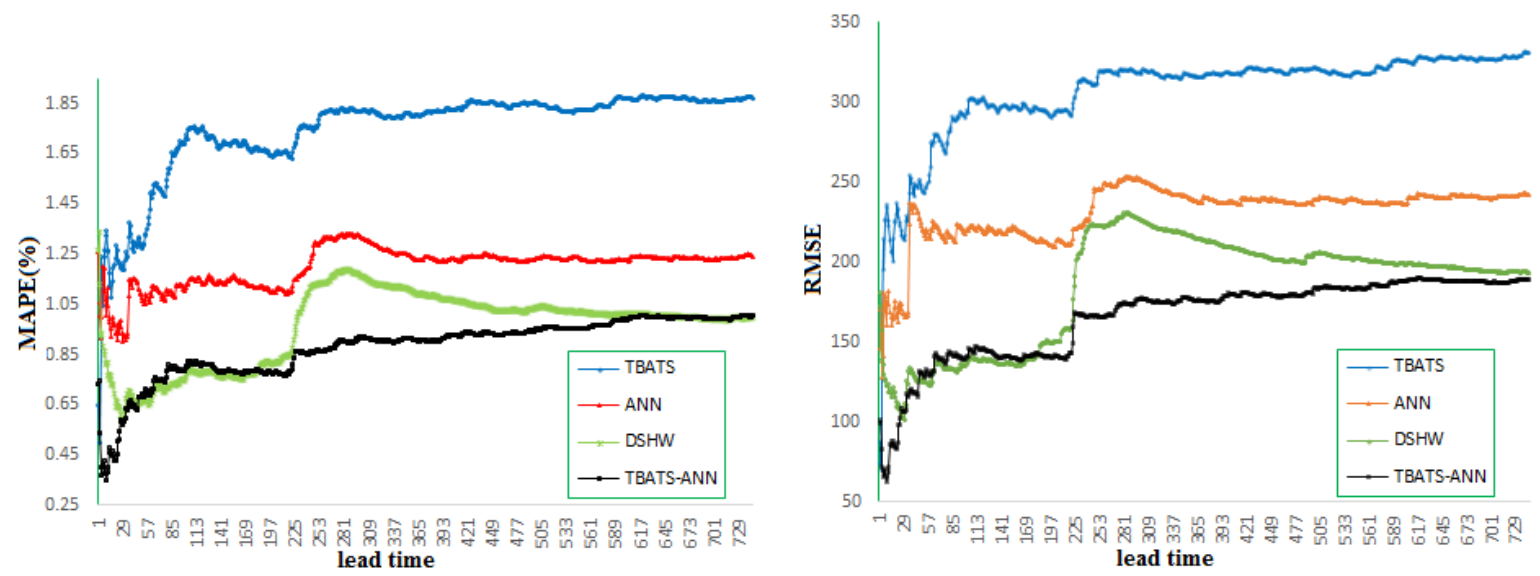

Fig. 3. Out-sample results of TBATS $((1,\{0,0\}, 0.812,\{\langle 24,11\rangle,\langle 168,6\rangle\})-\operatorname{ANN}(24,10,1)$, ANN(24-10-1), $\operatorname{DSHW}(24,168)$ and hybrid TBATS $(1,\{0,0\}, 0.812,\{<24,11\rangle,<168,6>\})-\mathrm{ANN}(24,10,1)$ for the load of Java Bali July 2010

\section{Conclusion}

This study proposed hybrid exponential smoothing state space and ANN to model the series with double seasonal patterns. The exponential smoothing state space named TBATS incorporates BoxCox transformation, ARMA error correction and trigonometric function with time varying based on Fourier. TBATS model is able to decompose and forecast time series with complex seasonal patterns. There is no guarantee that ARMA process appropriate to describe the irregular component of the series. This is probably caused by the existence of the nonlinearity pattern in the data that cannot handle by TBATS. ANN model is superior in handling nonlinear pattern. The hybrid TBATS-ANN is proposed to capture multiple seasonality, high frequency seasonality and nonlinearity pattern of the series therefore the forecast performance can be improved. Algorithm of the proposed hybrid approach is started by decomposing the load series into level, trend, seasonal and irregular components and continuing using the decomposed components as inputs of ANN. Thus the forecast can be obtained from ANN model with the inputs are the level, trend, seasonal and irregular components of the series. Based on the results for load data of Bawen and Java Bali substations which have integer and nested seasonal pattern, the proposed hybrid model generally yields smaller MAPE and RMSE than TBATS, ANN and DSHW.

\section{Acknowledgment}

We would like to thank the editor and anonymous reviewers for their valuable comments and suggestions. The comments and suggestions are very helpful for improving our manuscript.

\section{References}

[1] M. Bernardi, and L. Petrella, "Multiple seasonal cycles forecasting model: the Italian electricity demand," Stat Methods Appl, vol. 24, pp. 671-695, 2015.

[2] A. M. De Livera, R.J. Hyndman, and R. D. Snyder, "Forecasting time series with complex seasonal patterns using exponential smoothing. Journal of the American Statistical Association, vol. 106, issue 496, pp. 1513-1527, 2011.

[3] P. G, Gould, A. B. Koehler, J.K. Ord, R. D. Snyder, R.J. Hyndman, and F. Vahid-Araghi, "Forecasting time series with multiple seasonal patterns," European Journal of Operational Research, vol. 191, pp. 207$222,2008$. 
[4] O. B. Shukur, N. M. Fadhil, M. H. Lee, and M. H. Ahmad, "Electricity load forecasting using hybrid of multipilicative double seasonal exponential smoothing model with artificial neural network," Jurnal Teknologi, vol. 69, issue 2, pp. 65-70, 2014.

[5] Suhartono and A. J. Endharta, "Short term electricity load demand forecasting in Indonesia by using double seasonal recurrent neural network," International Journal of Mathematical Models and Methods in Applied Sciences, vol. 3, issue 3, pp. 171-178, 2009. http://www.naun.org/main/NAUN/ijmmas/19096.pdf

[6] J. W. Taylor, "Short-term electricity demand forecasting using double seasonal exponential smoothing," Journal of the Operational Research Society, vol. 54, pp. 799-805, 2003.

[7] P. R. Winter, "Forecasting sales by exponentially weighted moving averages," Management Science, vol. 6, issue 3, pp. 324-342, 1960.

[8] R. J. Hyndman, A. B. Koehler, J.K. Ord, and R. D. Snyder, Forecasting with exponential smoothing: the state space approach. Springer-Verlag, Berlin, 2008.

[9] J.K. Ord, A. B. Koehler, and R.D. Snyder, "Estimation and prediction for a class of dynamic nonlinear statistical models," Journal of the American Statistical Association, vol. 92, issue 440, pp. 1621-1629, 1997.

[10]R.J. Hyndman, A. B. Koehler, R.D. Snyder, and S. Grose, "A state space framework for automatic forecasting using exponential smoothing methods," International Journal of forecasting, vol. 18, issue 3, 439-454, 2002.

[11]J. W. Taylor, and R. D. Snyder, Forecasting intraday time series with multiple seasonal cycles using parsimonious seasonal exponential smoothing. Technical Report 09/09. Department of Econometrics and Business Statistics, Monash University, 2009.

[12]Haviluddin and A. Jawahir, " Comparing of ARIMA and RBFNN for short-term forecasting," International Journal of Advances in Intelligent Informatics, vol. 1, no.1, pp. 15-22, 2015.

[13] M. Yousefi, D. Hooshyar, A. R. Dooraki, K. S. M. Sahari, W. Khaksar, and F. B. I. Alnaimi, “ Short-term wind speed forecasting forecasting by an adaptive network-based fuzzy inference system (ANFIS): an attempt towards an ensemble forecasting method," International Journal of Advances in Intelligent Informatics, vol. 1, no.3, pp. 140-149, 2015.

[14]N. Gradojevic, "Non-linear, non-parametric, non-fundamental exchange rate forecasting," Journal of Forecasting, vol. 25, issue 4, pp. 227-245, 2006.

[15] A. Azadeh, S.F. Ghaderi, M. Sheikhalishahi, and B.P. Nokhandan, "Optimization of short load forecasting in electricity market of Iran using artificial neural networks," Optimization and Engineering, vol. 15, issue 2, pp. 485-508, 2014.

[16] A. I. Melhum, L. Omar, and S.A. Mahmood, "Short term load forecasting using artificial neural network," International Journal of Soft Computing and Engineering (IJCSE), vol. 3, issue 1, pp. 56-58, 2013.

[17]C. A. Moturi, and F. K. Kioko, F. K, "Use of artificial neural networks for short-term electricity load forecasting of Kenya National grid power system," International Journal of Computer Applications, vol. 63, issue 2, pp. 25-30, 2013.

[18]A. Shrivastava, and A. Bhandakkar, "Short term load forecasting using artificial neural network techniques," International Journal of Engineering Research and Applications, vol. 3, issue 5, pp. 1524$1527,2013$.

[19] S. Simsar, M. Alborzi, J. Nazemi, and M. A. Layyegh, "Forecasting power demand using neural networks model," International Journal of Engineering and Advanced Technology (IJEAT), vol. 2, issue 5, 441-446, 2013.

[20] J. W. Taylor, L. M. de Menezes, and P. E. McSharry, “A comparison of univariate methods for forecasting electricity demand up to a day ahead," International Journal of Forecasting, vol. 22, pp. 1-16, 2006.

[21] G. Dudek, “ Neural Networks for pattern-based short-term load forecasting: A comparative study," Neurocomputing, vol. 205, pp. 64-74, 2016.

[22]B. Dong, Z. Li, S.M.M. Rahman, and R. Vega, “ A hybrid approach for forecasting future residential electricity consumption," Energy and Buildings, vol. 117, pp. 341-351, 2016.

[23] H.J. Sadaei, R. Enayatifar, A. H. Abdullah, and A. Gani, "Short-term load forecasting using a hybrid model with a refined exponentially weighted fuzzy time series and an improved harmony search," Electrical Power and Energy System, vol. 62, pp. 118-129, 2014.

[24]W. Lee, and J. Hong, "A hybrid dynamic and fuzzy time series model for mid-term power load forecasting," Electrical Power and Energy Systems, vol. 64, pp. 1057-1062, 2015. 
[25] S. Gupta, V. Singh, A.P. Mittal, and A. Rani, "A hybrid modell of wavelet and neural network for short term load forecasting," International Journal of Electronic and Electrical Engineering, vol. 7, issue 4, pp. 387-394, 2014.

[26]C.C. Holt, "Forecasting seasonals and trends by exponentially weighted moving averages," International Journal of Forecasting, vol. 20, pp. 5-10, 2004

[27] A. Harvey, S. J. Koopman, and M. Riani, "The modelling and seasonal adjustment of weekly observations," Journal of Business \& Economic Statistics, vol. 15, issue 3, 354-368, 1997.

[28] A. Harvey, Forecasting structural time series models and the Kalman filter, Cambridge University Press, 1989.

[29]A. Lapedes, R. Farber, Nonlinear signal processing using neural networks: prediction and system modelling. Technical Report LA-UR-87-2662, Los Alamos National Laboratory, Los Alamos, NM, 1978.

[30]A. Lapedes, and R. Farber, How neural nets work. In: Anderson, D. Z., (Ed.), Neural Information Processing Systems, American Institute of Physics, New York, , 1988, pp. 442-456.

[31] G. Zhang, B. E. Patuwo, and M. Y. Hu, "Forecasting with artificial neural networks: The state of the art," International Journal of Forecasting, vol. 14, pp. 35-62, 1998.

[32] S. Sapna, A. Tailarasi, and M. P. Kumar, "Backpropagation learning algorithm based on Levenberg Marquardt Algorithm," Computer Science \& Information Technology, vol. 2, pp. 393-398, 2012. http://airccj.org/CSCP/vol2/csit2438.pdf 